\title{
Noise power scheduling in active noise control systems with online secondary path modeling
}

\author{
M. T. Akhtar ${ }^{1 a)}$, M. Abe Abd M. Kawamata $^{2}$ \\ ${ }^{1}$ Department of Electrical Engineering, UAE University, P.O.Box 17555, Al-Ain, \\ $U A E$ \\ ${ }^{2}$ Graduate School of Engineering, Tohoku University, Sendai 980-8579, JAPAN \\ a)akhtar@ieee.org
}

\begin{abstract}
In active noise control (ANC) systems, the online secondary path modeling (OSPM) methods that use additive random noise are often applied. The additive random noise, however, contributes to the residual noise, and thus deteriorates the noise control performance of ANC systems. This paper proposes a new OSPM method with power scheduling of additive random noise. Here the OSPM filter is adapted using a variable step size (VSS) LMS algorithm already proposed by authors. Furthermore, the additive-random-noise power is scheduled based on the convergence status of an ANC system. Computer simulations demonstrate the effectiveness of the proposed method.
\end{abstract}

Keywords: active noise control, FxLMS algorithm, secondary-path modeling, noise-power scheduling.

Classification: Science and engineering for electronics

\section{References}

[1] S. M. Kuo and D. R. Morgan, "Active Noise Control Systems-Algorithms and DSP Implementation," New York: Wiley, 1996.

[2] L. J. Eriksson and M. C. Allie, "Use of random noise for on-line transducer modeling in an adaptive active attenuation system," J. Acoust. Soc. Am., vol. 85, no. 2, pp. 797-802, 1989.

[3] C. Bao, P. Sas, and H. V. Brussel, "Adaptive active control of noise in 3-D reverberant enclosure," J. Sound Vibr., vol. 161, no. 3, pp. 501-514, 1993.

[4] S. M. Kuo and D. Vijayan, "A secondary path modeling technique for active noise control systems," IEEE Trans. Speech Audio Proc., vol. 5, no. 4, pp. 374-377, 1997.

[5] M. Zhang, H. Lan, and W. Ser, "Cross-updated active noise control system with online secondary path modeling," IEEE Trans. Speech Audio Proc., vol. 9, no. 5, pp. 598-602, 2001.

[6] M. Zhang, H. Lan, and W. Ser, "A robust online secondary path modeling method with auxiliurary power scheduling strategy and norm constraint manipulation," IEEE Trans. Speech Audio Proc., vol. 11, no. 1, pp. 4553, 2003.

[7] M. T. Akhtar, M. Abe, and M. Kawamata, "A New Variable Step Size 
LMS Algorithm-Based Method for Improved Online Secondary Path Modeling in Active Noise Control Systems," IEEE Trans. Audio Speech Lang. Proc., vol. 14, no. 2, pp. 720-726, 2006.

[8] M. T. Akhtar, M. Abe, and M. Kawamata, "A Technique for Active Noise Control Systems With Online Secondary Path Modeling Using Additive Noise Power Scheduling," IEICE Technical Report., vol. 105, no. 145, pp. 13-18, June 27, 2005.

\section{Introduction}

Active noise control (ANC) is based on the principle of destructive interference of propagating acoustic waves. The most popular adaptation algorithm used for ANC applications is the FxLMS algorithm [1]. The basic method for ANC systems with online secondary path modeling (OSPM) is proposed by Eriksson et. al [2]. As shown in Fig. 1 (a), here the reference signal, $x(n)$, is filtered through the model of the secondary path, $S(z)$, following the adaptive filter $W(z)$. The OSPM filter $\hat{S}(z)$ is used to model $S(z)$, and is updated using LMS algorithm as

$$
\hat{\boldsymbol{s}}(n+1)=\hat{\boldsymbol{s}}(n)+\mu_{s} f(n) \boldsymbol{v}(n)
$$

where $\mu_{s}$ is step size for $\hat{S}(z), f(n)=e(n)-\hat{v}^{\prime}(n)=\left[d(n)-y^{\prime}(n)\right]+\left[v^{\prime}(n)-\right.$ $\left.\hat{v}^{\prime}(n)\right]$ is the modeling-filter error signal, $d(n)=p(n) * x(n)$ is the primary noise, $y^{\prime}(n)=s(n) * y(n)$ is the canceling signal, $y(n)=w(n) * x(n)$ is output of $W(z), v^{\prime}(n)=s(n) * v(n)$ is the modeling signal, $\hat{v}^{\prime}(n)=\hat{s}(n) * v(n)$ is output of $\hat{S}(z)$ for $v(n), v(n)$ is additive random noise used for modeling, * denotes linear convolution, and $p(n), s(n)$, and $w(n)$ denote impulse response of $P(z), S(z)$ and $W(z)$, respectively.

The ANC filter $W(z)$ is updated using FxLMS algorithm as

$$
\boldsymbol{w}(n+1)=\boldsymbol{w}(n)+\mu_{w} g(n) \hat{\boldsymbol{x}}^{\prime}(n)
$$

where $\mu_{w}$ is step size for $W(z), g(n)=e(n)=\left[d(n)-y^{\prime}(n)\right]+v^{\prime}(n)$ is the control filter error signal, and $\hat{x}^{\prime}(n)=\hat{s}(n) * x(n)$ is reference signal $x(n)$ filtered through $\hat{S}(z)$.

In (1) and (2), $\left[d(n)-y^{\prime}(n)\right]$ is the desired error signal for adaptation of $W(z)$, but it acts as a disturbance for the adaptation of $\hat{S}(z)$. Due to this large disturbance, the convergence speed of $\hat{S}(z)$ is very slow, and it may diverge in a worst case. In order to improve the performance of Eriksson's method, various methods have been proposed in $[3,4,5,6]$. These methods add another third adaptive filter to the Eriksson's method. The Zhang's methods $[5,6]$ gives best performance among these methods.

In [7] we have proposed a two-adaptive-filter-based method for OSPM in ANC systems. Here we modify this method and propose using a variablepower additive random noise in the OSPM filter. The idea is to use initially a large signal for fast convergence of the modeling filter. Later, when ANC system converges the power of the additive random noise is reduced to a small 
value. The rest of the paper is organized as follows. Section 2 describes the proposed method. A few comments on computational complexity are also presented here. Section 3 presents the simulation results and discussion.

A version of this paper was presented at a domestic meeting and appeared in a technical report [8].

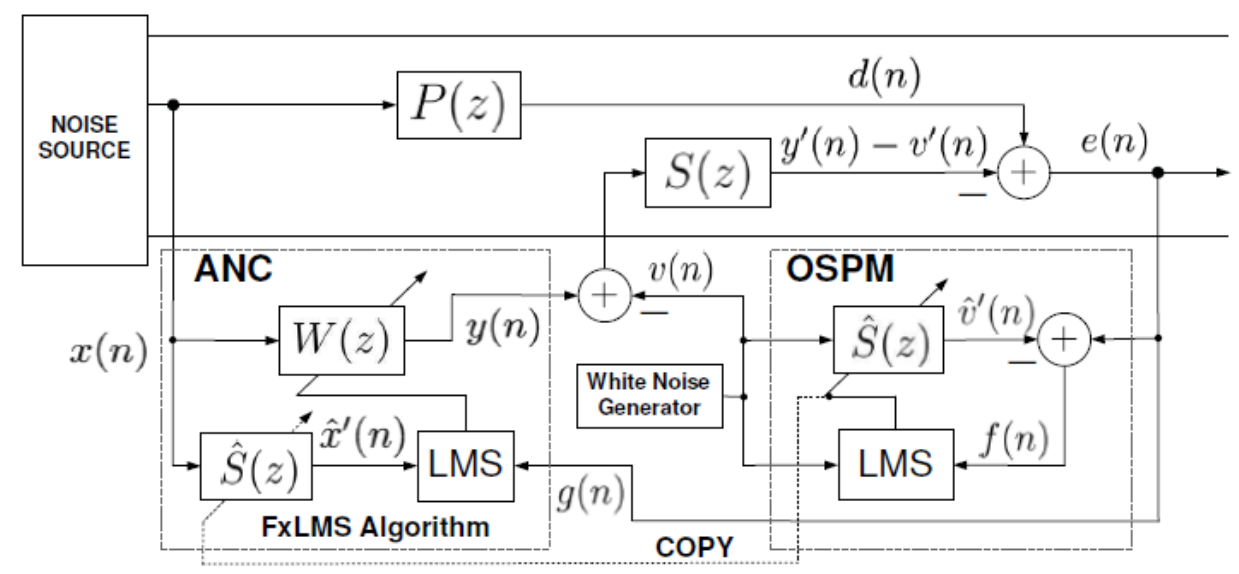

(a)

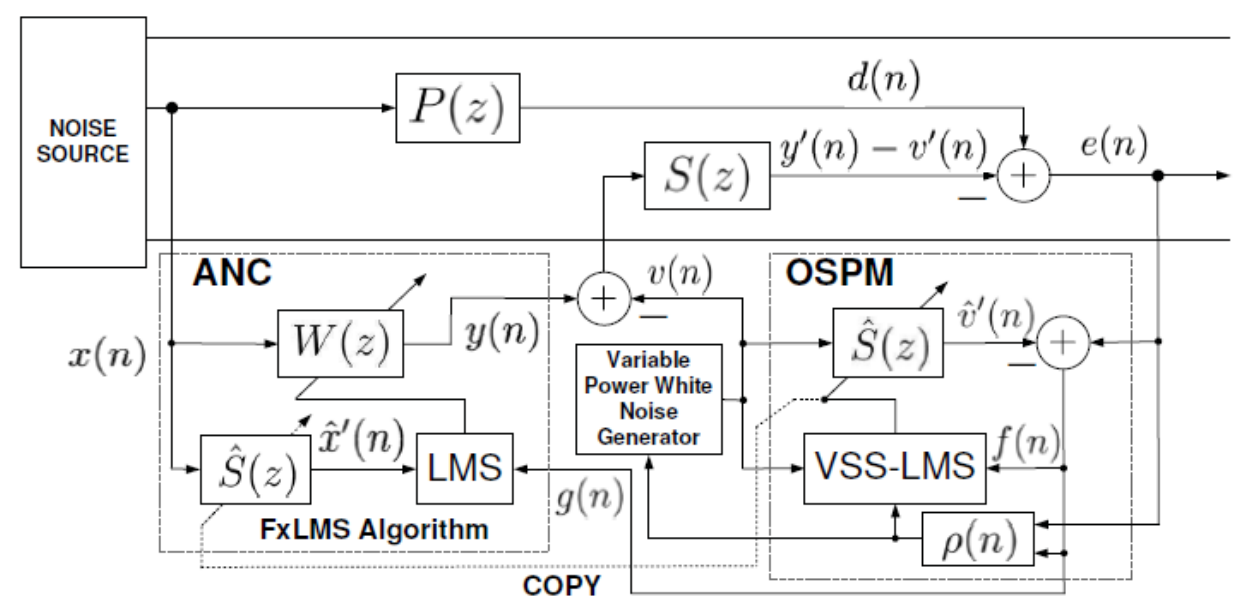

(b)

Fig. 1. ANC systems with online secondary path modeling (OSPM): a) Eriksson's method, b) Proposed method.

\section{Proposed method for ANC Systems With Online Secondary Path Modeling}

The proposed method is shown in Fig. 1 (b). We see that the basic structure of the proposed method is similar to Eriksson's method shown in Fig. 1 (a), except for the following modifications:

M.1. The OSPM filter $\hat{S}(z)$ is updated by variable step-size (VSS) LMS algorithm. The procedure to vary the step size is explained below: 
We define a ratio $\rho(n)=P_{f}(n) / P_{e}(n)$, where $P_{f}(n)$ and $P_{e}(n)$ are power of the modeling error signal $f(n)$ and the residual error signal $e(n)$, respectively. These powers can be estimated as

$$
P_{\gamma}(n)=\lambda P_{\gamma}(n-1)+(1-\lambda) \gamma^{2}(n)
$$

where $\lambda$ is the forgetting factor $(0.9<\lambda<1)$. A simple analysis shows that $\rho(0) \approx 1$ and $\lim _{n \rightarrow \infty} \rho(n) \rightarrow 0[7]$. Now the step size for $\hat{S}(z)$ is calculated as:

$$
\mu_{s}(n)=\rho(n) \mu_{s_{\min }}+(1-\rho(n)) \mu_{s_{\max }}
$$

where $\mu_{s_{\min }}$ and $\mu_{s_{\max }}$ are the experimentally determined values for lower and upper bounds of the step size. These values are selected so that neither adaptation is too slow nor it becomes unstable.

M.2. Both $W(z)$ and $\hat{S}(z)$ are adapted using same error signal $g(n)=$ $f(n)=e(n)-\hat{v}(n)[7]$.

M.3. The OSPM filter $\hat{S}(z)$ is excited by a variable power white noise generator.

The method based on modifications M.1. and M.2. was proposed by authors in [7]. The method showed a best OSPM performance from those considered in [7]. In order to further improve the performance of this method, here we propose to varying the power of the additive random noise $v(n)$ (modification M.3.).

We have already found a parameter $\rho(n)$ which can track variations in $\left[d(n)-y^{\prime}(n)\right]$, and hence can indicate the convergence status of the ANC system: $\rho(n) \approx 1 \Rightarrow\left[d(n)-y^{\prime}(n)\right]$ is large, and $\rho(\infty) \rightarrow 0 \Rightarrow$ ANC system has converged and $\left[d(n)-y^{\prime}(n)\right]$ is reduced. Similar to (4), the variance of the additive noise $v(n)$ can be adjusted as follows:

$$
v(n)=\sqrt{(1-\rho(n)) \sigma_{v_{\min }^{2}}^{2}+\rho(n) \sigma_{v_{\max }^{2}}^{2}} \cdot v_{m}(n)
$$

where $\sigma_{v_{\min }}^{2}$ and $\sigma_{v_{\max }}^{2}$ are minimum and maximum values for the variance of $v(n)$, respectively, and $v_{m}(n)$ is the white random noise of unity variance. In comparison with the three-adaptive-filter-based Zhang's methods $[5,6]$, the main features of the proposed method are:

1. It comprises only two adaptive filters and hence has reduced computational complexity. This is advantageous, especially when dealing with the long adaptive filters, which is usually the case in ANC.

2. By choosing appropriate values for $\sigma_{v_{\min }}^{2}$ and $\sigma_{v_{\max }}^{2}$, it gives better control over the variance of the additive random noise $v(n)$. The minimum variance can be selected so that the modeling filter can track small variations in steady state. 


\section{Simulation results and discussion}

This section presents the simulation experiments performed to verify the effectiveness of the proposed method. The following methods are considered: 1) Zhang's method-a [5], 2) Zhang's method-b [6] (a modified version of Zhang's method-a), 3) Previous method [7] (a method previously proposed by authors), and 4) Proposed method (a modified version of the Previous method, and is described in this paper). The performance comparison is done on the basis of the relative modeling error, being defined as $\Delta S(n)=$ $(\|\hat{\boldsymbol{s}}(n)-\boldsymbol{s}(n)\| /\|\boldsymbol{s}(n)\|)$.

The acoustic paths and all adaptive filters are modeled as FIR filters of tap-weight lengths, as given in Table I. For the primary and secondary paths the experimental data provided by [1] is used. The ANC filter $W(z)$, and the third filter $H(z)$ in Zhang's method, are initialized by null vectors of appropriate orders. To initialize $\hat{S}(z)$, offline secondary path modeling is performed which is stopped when the modeling error has been reduced to $-5 \mathrm{~dB}$. A sampling frequency of $4 \mathrm{kHz}$ is used. The reference signal $x(n)$ is a narrowband signal comprising frequencies of $50 \mathrm{~Hz}, 100 \mathrm{~Hz}, 200 \mathrm{~Hz}$ and $300 \mathrm{~Hz}$. Its variance is adjusted to 2 and a zero-mean white noise is added with SNR of $30 \mathrm{~dB}$. In Zhang's method-a and in previous method, the modeling excitation signal $v(n)$ is a zero-mean white Gaussian noise of constant variance 0.05 . The step size parameters for various adaptive filters are adjusted by trial-and-error, for fast and stable convergence, and are summarized in Table I. All the results shown below are averaged over 10 experiments.

Table I. Simulation parameters for computer experiments.

\begin{tabular}{|c|c|c|c|c|}
\hline \multicolumn{5}{|c|}{ Acoustic Paths and Adaptive Filters } \\
\hline FIR filter: & $P(z) \quad S(z)$ & $W(z)$ & $\hat{S}(z)$ & $H(z)$ \\
\hline Tap-weight length: & 128 & 64 & 64 & 48 \\
\hline \multicolumn{5}{|c|}{ Step-size and other parameters } \\
\hline Eriksson's methods: & \multirow{2}{*}{\multicolumn{4}{|c|}{$\begin{array}{l}\mu_{w}=5 \times 10^{-5}, \mu_{s}=1 \times 10^{-3} \\
\mu_{w}=5 \times 10^{-5}, \mu_{h}=2 \times 10^{-3}, \Delta=30 \\
\mu_{s}(\text { method-a })=1 \times 10^{-2}\end{array}$}} \\
\hline Zhang's methods: & & & & \\
\hline Proposed method: & \multicolumn{4}{|c|}{$\begin{array}{l}c(\text { method-b })=1.5, \mu_{s}(\text { method-b })=2 \times 10^{-3} \\
\mu_{w}=5 \times 10^{-5}, \sigma_{v_{\max }}^{2}=1.0, \sigma_{v_{\min }}^{2}=0.001 \\
\mu_{s_{\min }}=1 \times 10^{-3}, \mu_{s_{\max }}=1 \times 10^{-2} .\end{array}$} \\
\hline
\end{tabular}

Figure $2(\mathrm{a})$ shows the curves for relative modeling error, $\Delta S(\mathrm{~dB})$, for various methods (where previous method is authors' previous work [7]). Here, at $n=50,000$, the acoustic paths change. The Zhang's method-b gives a fast initial convergence, but higher steady state value. This degraded modeling performance is due to the fact that the variance of the modeling excitation signal $v(n)$ reduces to a very low level. We see that the proposed method gives best secondary path modeling performance in both the convergence speed and steady state mismatch. The corresponding curves for residual 
noise are shown in Fig. 2 (b).

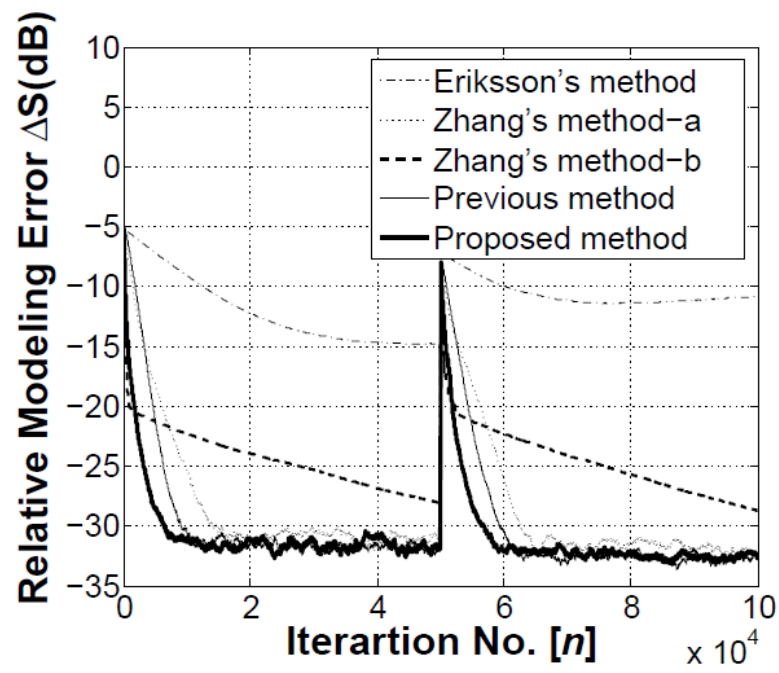

(a)

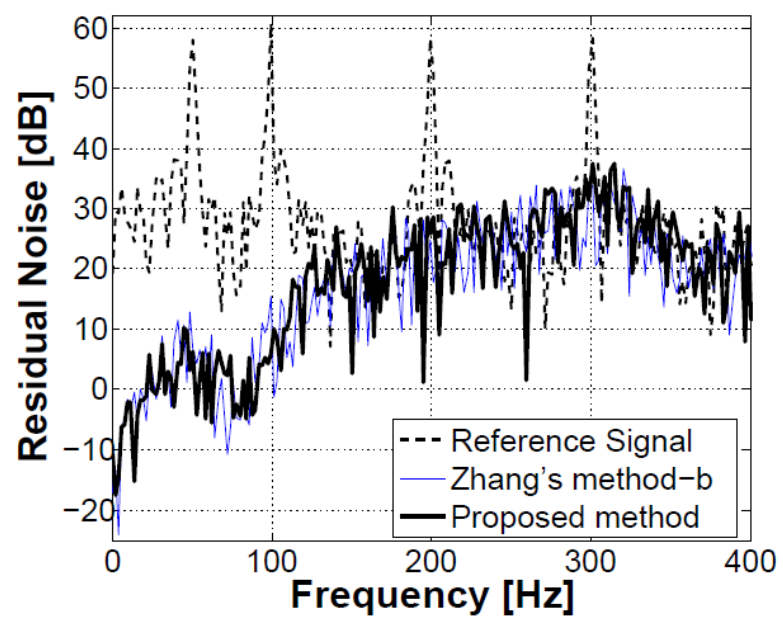

(b)

Fig. 2. Simulation results: a) Variation of the relative modeling error $\Delta S(n)$, b) Residual noise in steady state.

\section{Conclusion}

In this letter we have proposed a new method for OSPM in ANC systems. The structure of the proposed method is similar to the basic method [2], and it performs better than the Zhang's method [6], which comprises three adaptive filter. This improved OSPM performance is archived at no compromise to the noise reduction performance. 